\title{
FOCUS: PATIENT SAFETY AND THE MEDICAL LABORATORT Laboratory Using the IOM Aims
}

\section{CATHERINE N OTTO}

\section{LEARNING OBJECIVES}

1. Identify steps in the total testing process at the greatest risk for errors.

2. Explain the relationship of evidence-based management to evidence-based medicine.

3. Discuss the difference between patient-centered and system-focused healthcare.

4. Define timeliness with respect to patient-centered care.

5. Differentiate efficient and effective laboratory testing services.

6. Identify methods to provide equitable laboratory services.

ABBREVIATIONS: ADA, American Diabetes Association; AIC, hemoglobin $A_{1 C}$; EBM, evidencebased medicine; EBMgt, evidence-based management; $\mathrm{ED}$, emergency department; EHR, electronic health record; LOS, length of stay; POCT, point-of-care testing; T4, thyroxine; TAT, turnaround time; TSH, thyroid stimulating hormone; TTP, total testing process.

INDEX TERMS: Quality Improvement; Healthcare Quality Assurance

Clin Lab Sci 2011;24(2):108

Catherine N. Otto, PhD, MLS(ASCP) ${ }^{C M}$, SH(ASCP $)^{C M}$, $D L M(A S C P)^{C M}$, Portland, Oregon.

Address for Correspondence: Catherine N. Otto, PhD, $M L S(A S C P)^{C M}, S H(A S C P)^{C M}, D L M(A S C P)^{C M}$, Portland, Oregon,catherineotto@q.com

All healthcare should be safe, effective, patient-centered, timely, efficient, and equitable. ${ }^{1}$ The Institute of Medicine's (IOM) recommendations for improving healthcare quality create significant opportunities for medical laboratory professionals to improve patients' outcomes and to illustrate the quality of medical laboratory testing services to others.

\section{Safe Laboratory Services}

Medical laboratory services should provide care that improves patient outcomes. Errors or defects can occur at any step of the laboratory testing process. The majority of errors are identified in the pre-analytic ( 46.0 - 68.2\%) and post-analytic $(18.5-47.0 \%)^{2}$ phases. Error rate benchmarks are difficult to calculate because no standard mechanism exists to report errors for any phase of laboratory testing. Studies have reported error rate metrics as errors per sample, ${ }^{3}$ per analyses, ${ }^{4}$ per requisition ${ }^{5}$ and per billable test. ${ }^{6}$ Nonetheless, measuring error rates, followed by process or technology enhancements, ameliorates system problems, improves the total testing process (TTP), and enhances patient outcomes. ${ }^{3,4,7}$

The first and last steps in the TTP, 'selecting the correct test to perform' and 'accurately interpreting test results' are at the greatest risk for errors. ${ }^{8}$ Guidelines for test selection and reflexive testing protocols reduces the number of test ordering errors by decreasing unnecessary and ineffective test utilization. ${ }^{9}$ Examples of effective use of reflexive testing includes performing factor assays if a mixing study indicates a possible factor deficiency; performing antibody identification following a positive antibody screen in transfusion services; and performing a thyroxine (T4) if the thyroid stimulating hormone (TSH) is greater than an established threshold. ${ }^{9}$

An estimated $5 \%$ of medical errors are related to misinterpretation of laboratory test results. ${ }^{10}$ Interpretation of many laboratory tests requires more 


\section{FOCUS: PATIENT SAFETY AND THE MEDICAL LABORATORY}

information than a quantitative value and its place within a reference range. Often clinicians need an explanation. Laboratory-supplied interpretations of test results provide valuable information for the clinician particularly for new or complex tests such as special coagulation, serum protein electrophoresis and for circumstances related to specimen integrity. ${ }^{11}$ Special coagulation test interpretations reduced misdiagnosis and improved timeliness for diagnosis in approximately $75 \%$ of cases. ${ }^{12}$ Another area that creates confusion when interpreting test results occurs when comments are attached indicating compromised specimen integrity. Only $30 \%$ of residents were confident interpreting test results reported with comments regarding the presence of hemolysis. ${ }^{13}$

Improving the safety of laboratory testing services requires using a consistent data collection metric and focusing upon the first and last steps of the TTP. Laboratory professionals need to collaborate with clinicians to develop testing protocols and encourage requests for interpretations.

\section{Effective Laboratory Services}

Effectiveness is a fundamental healthcare delivery system requirement, and evidence is critical to establish appropriate use of laboratory tests. Measuring effectiveness of medical laboratory services is complicated by the varied test purposes: screening, diagnosis, prognosis, and monitoring of treatment. Characteristics of laboratory tests differ in sensitivity, specificity, and positive and negative predictive values. ${ }^{14}$ Although each TTP step is important, the first 'selecting the correct test to perform' and the last 'accurately interpreting test results' are the most critical to ensure effective laboratory services.

Improving effectiveness requires more than identifying which laboratory test or testing protocol best identifies a condition or monitors therapy; it also requires understanding how to effectively implement the protocol for its use in diverse healthcare settings. Evidence-based medicine (EBM) identifies best practices for providing medical care. Best practices, also called practice guidelines, are based upon scientific evidence, and developed by professional associations, accrediting organizations and government-sponsored groups. Successful implementation of best practices in healthcare settings requires evidence-based management (EBMgt) that addresses organizational structure, human behaviors and change management. ${ }^{15}$ EBMgt examines features of organizations that influence how care is delivered using principles from social and behavioral sciences and health services research. These factors and employing EBMgt principles are critical components of using quality improvement processes, such as PDSA (plan-do-study-act). ${ }^{16}$

Employing EBM and EBMgt for highly prevalent conditions can demonstrate effectiveness using a process improvement model. The American Diabetes Association (ADA) recommends performing hemoglobin $A_{1 C}(A 1 C)$ biannually for patients to meet treatment goals and quarterly for those who do not. ${ }^{17}$ Managing patients with diabetes, a chronic disease, requires an infrastructure and procedures to monitor and follow patients over long periods of time. Information systems are a highly beneficial component of patient management infrastructures. An automated patient reminder protocol raised semiannual test compliance and maintained better glycemic control. ${ }^{18}$ Important EBMgt principles to consider when implementing a project of this nature include size of the organization-large organizations often have greater access to technology; availability of established patient reminder programs - is the new program compatible with the established program; and adaptability to change-incentives for providers and patients to change behaviors.

Identifying an appropriate testing and timing protocol for heparin therapy is another example of employing EBM and EBMgt principles to ensure effectiveness. Coagulation testing is essential for monitoring patients on standard-dose unfractionated heparin therapy in order to achieve therapeutic levels of anticoagulation and reduce risks of supratherapeutic levels that may lead to hemorrhage. One heparin monitoring protocol recommends all patients receive activated partial thromboplastin time (aPTT) testing within 12 hours of initiation of therapy, all patients receive platelet counts within 72 hours of therapy initiation, $90 \%$ of patients achieve therapeutic anticoagulation levels within 24 hours and $75 \%$ of patients have no more than 1 


\section{FOCUS: PATIENT SAFETY AND THE MEDICAL LABORATORY}

supratherapeutic level of anticoagulation during the first 72 hours of therapy. ${ }^{19}$ Implementing this type of protocol requires working together with clinicians, nurses and pharmacists to identify specific tests to be performed and their timing to monitor anticoagulation status, as well as their respective responsibilities.

\section{Patient-Centered Laboratory Services}

Respecting patient preferences, recognizing cultural differences and educating patients about medical laboratory tests is a primary component of providing patient-centered healthcare. Unlike many of the IOM recommendations for improving healthcare delivery, "patient-centered care is not a check-list, dashboard or an action plan. ${ }^{20}$ It requires viewing the provision of laboratory services from the perspective of the patient while employing care that is safe and of high clinical quality. Patient-centered approach requires medical laboratory professionals to educate patients about laboratory tests ordered as part of their medical care. This can be accomplished by providing instructions to patients prior to specimen collection, such as fasting requirements for a lipid profile or glucose test ${ }^{21}$ and tips on how to care for the venipuncture site. ${ }^{22}$ Instruction may take place in a face-to-face format or by supplying instructional tools for laboratory practitioners, clinicians and nursing staff to distribute to patients. ${ }^{21-23}$ Instructional and educational tools must be at an appropriate literacy level and in other languages for non-English speakers to be effective. ${ }^{23}$ Providing instructional materials to patients is appropriate patientcentered care and quality medical laboratory services.

Healthcare delivery needs a cultural transformation to become patient-centered. ${ }^{20}$ One method to become patient-centered is to use patients instead of specimens to measure laboratory productivity. Because laboratory productivity is based upon numbers of tests performed, it communicates what is valued. This is understandable given the reimbursement mechanism in place. However, without changing the reimbursement system, the number of patients served on a daily, weekly, or monthly basis can be monitored and shared with staff and administration. Two methods to do this are calculating the number of tests performed per patient encounter for the outpatient setting or number of laboratory tests performed per 1000 patient days.

\section{Timely Laboratory Services}

Improving patients' lives requires laboratory test results to be performed and reported to clinicians in a timeframe that does not delay the next step in their care process. Timeliness or turnaround time (TAT) is not a new concept for laboratorians; its measurement is a significant component of quality assessment for medical laboratory services. However, no standard metric exists. Does it begin when a specimen is collected and end when the test result is reported? - a laboratory-centered focus. Or does it begin when the clinician orders the test and ends when action is taken by the clinician?- - a patient-centered focus. Patient-centered care will be enhanced if timeliness is considered from the perspective of the entire healthcare system.

Physicians are often dissatisfied with laboratory TAT. ${ }^{24,}{ }^{25}$ Understanding and examining how laboratory timeliness impacts other healthcare services is important to improving quality. Timely laboratory test results are important for patients in surgery, intensive care units and emergency department (ED). Consequences of delayed or increased laboratory test TAT for the ED are longer patient wait times, delay in follow-up testing, ambulance diversion and patient dissatisfaction. ${ }^{26}$ Point-of-care testing (POCT) is frequently considered as an option to decrease laboratory testing TAT for the ED. Analytical modeling using simulations is a tool to examine implementing new programs, such as comparing a POCT program with existing medical laboratory services. Modeling uses actual data to predict outcomes based upon varying assumptions. For example, if POCT reduced ED laboratory TAT from 120 to 10 minutes, ED length of stay (LOS) would be reduced from 2.77 to 2.17 hours, an improvement of 26 minutes. ${ }^{26}$ However, if POCT reduced ED TAT from 60 minutes to 10 minutes, the reduction of ED LOS is only 0.1 hours, an improvement of 6 minutes. ${ }^{26}$ Other considerations may be factored into the simulation such as increasing the number of patients seen per day, or decreasing the number of patients diverted due to overcrowding in the ED. Simulation modeling is a viable analytical tool to examine options to improve processes in healthcare delivery, when used with institution data and quantitative assumptions. 


\section{FOCUS: PATIENT SAFETY AND THE MEDICAL LABORATORY}

Treatment delays often occur for discharged patients who have pending laboratory tests if there is no formal mechanism to communicate with the primary care physician. ${ }^{27,28}$ An examination of pending laboratory tests in two academic tertiary care centers revealed $41 \%$ of discharged patients had pending diagnostic tests, most of which were microbiological analyses. ${ }^{28}$ Approximately $16 \%$ of all discharged patients had test results requiring clinician action. Solutions for this breakdown in the TTP require more than a laboratory process intervention. It requires examining the situation, by collecting data and then establishing an interdepartmental process improvement team to employ EBMt principles to identify and implement solutions. Possible solutions include identifying pending tests on discharge summaries and sending discharge summaries to primary care physicians. ${ }^{27}$

Timely laboratory testing services is best considered from the perspective of the user of laboratory test information-the clinician and the recipient of the care- the patient. Laboratory professionals can improve timeliness of testing services by seeking opportunities to work with other healthcare professionals.

\section{Efficient Laboratory Services}

Efficient laboratory services avoid and reduce waste. Any process that must be repeated is inefficient. Mislabeled, unlabeled, and lost specimens are examples of inefficiency because each event requires recollecting the specimen-a waste of personnel time and supplies resulting in a delay of patient care. Specimens that are hemolyzed, clotted, or insufficient quantity also contribute to the inefficiency of laboratory services. These inefficiencies are identified as errors or defects in the laboratory testing process. Although most of these errors do not result in harm to the patient, their presence creates waste in the laboratory testing process which leads to increased costs and delays in patients' care. $^{29}$

Measuring deviations in the steps of the TTP identifies opportunities to reduce waste. Error rates may be reported as a per label, percentage, per billable test or per specimen: 0.92 errors per 1000 labels, ${ }^{3}$ 1.0\% mislabeled samples, ${ }^{3}$ requisition mismatches $(6.3 \%)^{3}$ requisition mismatches and unlabeled $4.6 \%{ }^{3}$ unlabeled samples, and 55 results reported for the wrong specimen per 1,000,000 billable tests. ${ }^{6}$ It is preferable to report all deviation in the TTP (pre-analytical, analytical, postanalytical) in a consistent manner, i.e. using an identical denominator to compute error rates. Although defects identified in the analytical phase are different than those found in the post-analytic phase, using one denominator to compute error rates measures efficiency of the TTP. Error rates can be calculated for each of the three testing phases as well as for each step in the TTP. Categories of deviations may also be used for steps such as specimen collection in order to quantify specific deviations such as mislabeled and unlabeled specimens. Data such as error rates must also be linked to their effects upon the healthcare delivery system, such as increased cost and delays to patient care, in order to calculate the effects of the inefficiencies.

\section{Equitable Laboratory Services}

Ensuring the equity of medical laboratory services requires that there is no variation in the provision of services due to gender, ethnicity, geographic location and socioeconomic status. Providing equitable medical care is also reflected in policies relating to access to services-health insurance, availability of primary care clinicians and specialty services-factors that cannot be ameliorated by laboratory policies. However, there are features of equity with respect to patients' access to laboratory services that laboratory practitioners can evaluate and rectify: Are specimen collection stations available at times and in convenient locations for the patient population? Are translation services or bilingual employees available to patients who need these services? Are patient education materials provided for these same patients?22 The primary focus of this aim is determining if medical laboratory services are provided in a fair manner to all.

Components of patient-centered care are also reflected in providing equitable laboratory testing services; specifically that of providing care based upon individual needs. Supplying instructional material at an appropriate literacy level and for non-English speakers is a feature of providing equitable laboratory services.

\section{SUMMARY}

Examining the quality of medical laboratory services us- 


\section{FOCUS: PATIENT SAFETY AND THE MEDICAL LABORATORY}

ing the perspective of the six IOM aims provides laboratory professionals with numerous opportunities to improve the quality of healthcare and validate the value of medical laboratory test information in improving patients' lives. This necessitates exploring the effects of other healthcare processes upon the TTP, as well as the affect laboratory test information and processes have upon other healthcare delivery processes. It requires using analytical approaches that value data collection, systematic process improvement models and lessons learned in other disciplines.

\section{REFERENCES}

1. Institute of Medicine. Crossing the Quality Chasm: A new health system for the $21^{\text {st }}$ century. Washington, DC: National Academic Press; 2001. 23-60.

2. Plebani M. Errors in clinical laboratories or errors in clinical laboratory medicine? Clin Chem Lab Med 2006; 44:750-9.

3. Wagar EA, Tamashiro L, Yasin B, Hilborne L, Bruckner DA. Patient safety in the clinical Laboratory, a longitudinal analysis of specimen identification errors. Arch Pathol Lab Med. 2006; 130;1662-8.

4. Carraro P, Plebani M. Errors in a stat laboratory: types and frequencies 10 years later. Clin Chem 2007;53:1338-42.

5. Wang S, Ho V. Corrections of clinical laboratory test results in a laboratory information system. Arch Pathol Lab Med 2004; 128:890-2.

6. Valenstein PN, Raab SS, Walsh MK. Identification errors involving clinical laboratories, a College of American Pathologists Q-probes study of patient and specimen identification errors at 120 institutions. Arch Pathol Lab Med 2006;130:1106-13.

7. Howanitz PJ, Renner SW, Walsh MK. Continuous wristband monitoring over 2 years decreases identification errors, a College of American Pathologists Q-tracks study. Arch Pathol Lab Med 2002;126:809-15.

8. Plebani M. Exploring the iceberg of errors in laboratory medicine. Clin Chim Acta 2009; 404:16-23.

9. Laposata M, Dighe A. Pre-pre and post-post analytical error: high-incidence patient safety hazards involving the clinical laboratory. Clin Chem Lab Med 2007;45:712-9.

10. Piva E, Plebani M. Interpretive reports and critical values. Clin Chem Acta 2009;404:52-8.

11. Plebani M. Interpretive commenting: a tool for improving the laboratory-clinical interface. Clin Chim Acta 2009;404:46-51.

12. Laposata ME, Laposata M, Van Cott EM, Buchner DS, Kashalo MS, Dighe AS. Physician survey of a laboratory medicine interpretive service and evaluation of the influence of interpretations on laboratory test ordering. Arch Pathol Lab Med 2004;128:1424-7.

13. Stanfliet JC, Maccauley J, Pillay TS. Quality of teaching in chemical pathology: ability of interns to order and interpret laboratory tests. J Clin Pathol 2009;62:664-6.
14. Otto CN. Screening protocols for diseases in women: a review of screening principles and examination of practices. Clin Lab Sci 1999;12:230-8.

15. Shortell SM, Rundall TG, Hsu J. Improving patient care by linking evidence-based medicine and evidence-based management. JAMA 2007;298:673-6.

16. Berwick DM. Broadening the view of evidence-based medicine. Qual Saf Health Care 2005;14(5):315-6.

17. American Diabetes Association. Executive Summary: Standards of medical care in diabetes-2011. Diabetes Care 2011; 34(Supp 1): S4-S10.

18. Valenstein PN, Walsh MK, Pappas AA, Howanitz PJ. Interinstitutional variation in glycohemoglobin monitoring and glycemic control in diabetic patients. Arch Pathol Lab Med 2001;125:191-7.

19. Valenstein PN, Walsh MK, Meier F. Heparin monitoring and patient safety, a College of American Pathologists Q-probes study of 3431 patients at 140 institutions. Arch Pathol Lab Med 2004;128:397-402.

20. Frampton S, Guastello S, Brady C, Hale M, Horowitz S, et al. Patient-Centered Care Improvement Guide. Derby, Connecticut: Planetree; October 2008. Available from: http://www.ihi.org/

IHI/Topics/PatientCenteredCare/PatientCenteredCareGeneral /Tools/PatientCenteredCareImprovementGuide.htm Accessed 2011 January 5. 1-20.

21. Patient Safety Committee. ASCLS. Fasting Patient Safety Tips-English version. Available from : http://www.ascls.org/ leadership/psc/Fasting\%20Patient\%20Safety\%20Tips.pdf Accessed 2011 January 5.

22. Patient Safety Committee. ASCLS. Venipuncture Patient Safety Tips. Available from: http://www.ascls.org/leadership/ psc/Venipuncture\%20Patient\%20Safety\%20Tips.pdf Accessed 2011 January 5.

23. Patient Safety Committee. ASCLS. Fasting Patient Safety Tips-Spanish version. Available from: http://www.ascls.org/ leadership/psc/Spanish\%20Fasting\%20Patient\%20Safety\%20 Tips.pdf Accessed 2011 January 5.

24. Jones BA, Bekeris LG, Nakhleh RE, Walsh MK, Valenstein PN. Physician satisfaction with clinical laboratory services, a College of American Pathologists Q-probes study of 138 institutions. Arch Pathol Lab Med 2009;133:38-43.

25. Steindel SJ, Howanitz PJ. Physician satisfaction and emergency department laboratory test turnaround time, observations based on College of American Pathologists Q-probes studies. Arch Pathol Lab Med 2001;125:863-71.

26. Storrow AB, Zhou C, Gaddis G, Han JH, Miller K, et al. Decreasing lab turnaround time improves emergency department throughput and decreases emergency medical services diversion: a simulation model. Acad Emerg Med 2008;15:1130-5.

27. Kripalani S, LeFevre F, Phillips CO, Williams MV, Basaviah P, et al. Deficits in communication and information transfer between hospital-based and primary care physicians, implications for patient safety and continuity of care. JAMA 2007;297:831-41. 
28. Roy CL, Poon EG, Karson AS, Ladak-Merchant, Z, Johnson $\mathrm{RE}$, et al. Patient safety concerns arising from test results that return after hospital discharge. Ann Intern Med 2005;143:1218.
29. Bonini P, Plebani M, Ceriotti F, Rubboli F. Errors in laboratory medicine. Clin Chem 2002;48:691-8.

30. Wagar EA, Stankovic AK, Raab S, Nakhleh RE, Walsh MK. Specimen labeling errors, a Q-probes analysis of 147 clinical laboratories. Arch Pathol Lab Med 2008;132:1617-22.

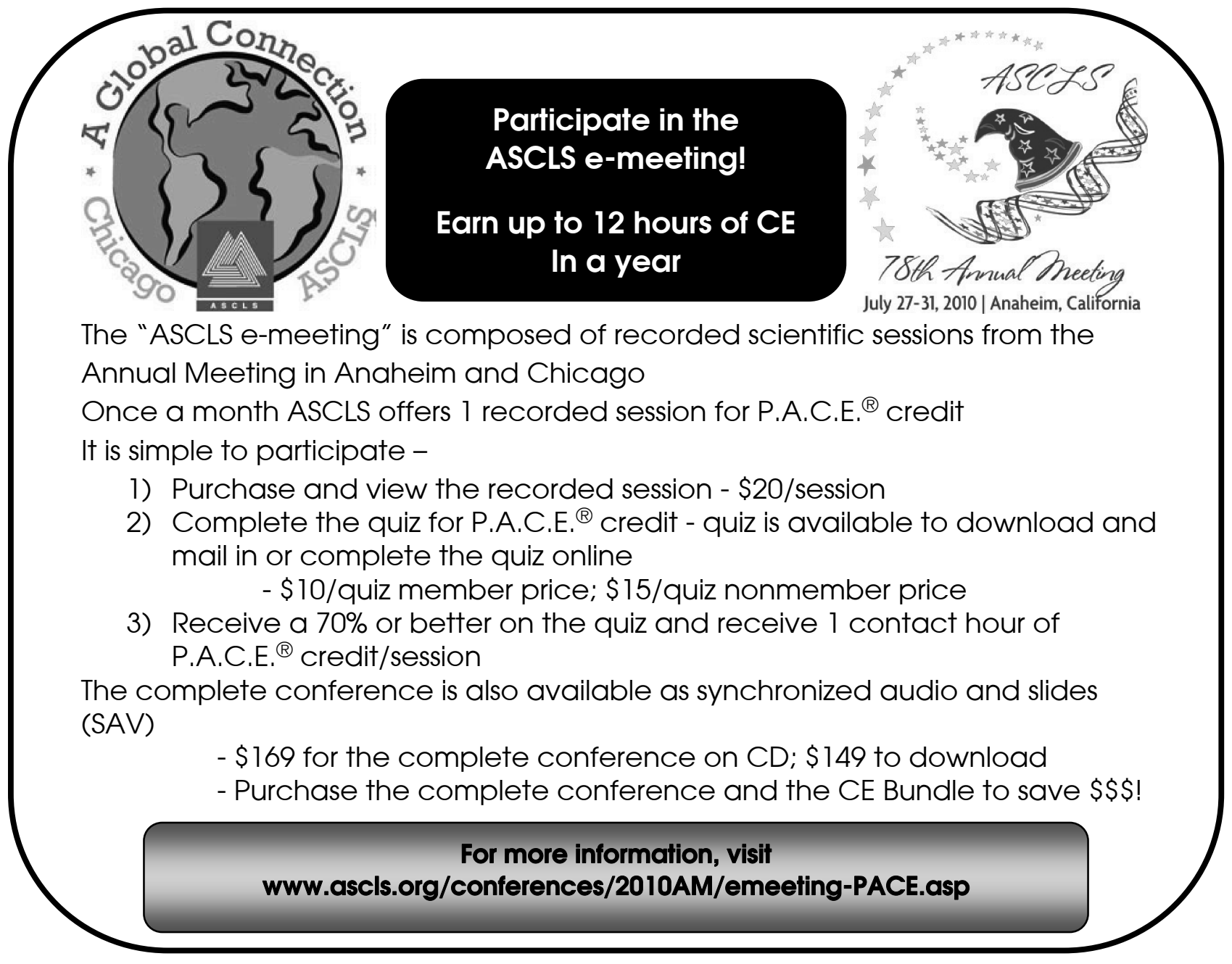

